\title{
Informe pericial de un caso de choque de embarcación contra un muelle en Paraguay
}

\author{
P. G. Yugovich ${ }^{1}$, G. A. Melgarejo ${ }^{1}$
}

${ }^{1}$ Facultad de Ciencias y Tecnología - Universidad Católica "Nuestra Sra. de la Asunción. Tte. Cantaluppi y G. Molinas, Campus Barrio Santa Librada, Asunción, Paraguay.

\section{Información del artículo \\ DOI: \\ http://dx.doi.org/10.21041/ra.v $\underline{1 i 2.11}$ \\ Artículo recibido el 03 de Diciembre de 2010, revisado bajo las políticas de publicación de la Revista ALCONPAT y aceptado el 03 de Marzo de 2011. Cualquier discusión, incluyendo la réplica de los autores se publicará en el primer número del año 2012 siempre y cuando la información se reciba antes del cierre del tercer número del año 2011}

\section{(C) 2011 Alconpat Internacional}

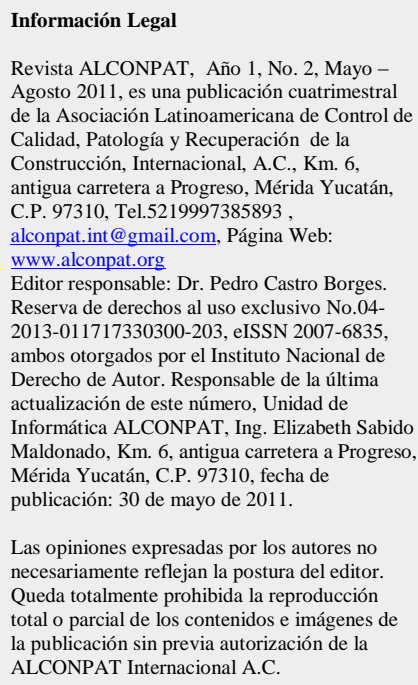

\section{RESUMEN}

Un muelle que facilita la descarga de GLP (gas licuado de petróleo) en la ciudad de San Antonio, Paraguay, fue impactado por una barcaza en proceso de maniobras. Se produjo la fractura del elemento impactado (un pilar) y aparecen otros síntomas, como fisuras y deformaciones, en otros elementos contiguos. La propietaria del muelle reclama a la aseguradora un siniestro total, amparada en los síntomas que se observan en toda la estructura del muelle. La pericia realizada por la liquidadora de siniestros requirió la participación de un profesional de la ingeniería con conocimientos de materiales, estructuras y patología de las construcciones. El objetivo del informe es determinar el alcance real del choque, con sus probables efectos sobre el resto de la estructura, acotando las consecuencias del siniestro y por ende, la responsabilidad de la aseguradora. El estudio incluye el diagnóstico y la recomendación de acciones necesarias para la reparación, refuerzo y mantenimiento de la estructura. El diagnóstico determina, en lo sustancial, que solo una parte de los síntomas son atribuibles al choque, siendo que la estructura presenta otros problemas que no se pueden atribuir al mismo.

Palabras clave: patología; estructuras; pericia; choque; durabilidad.

\section{ABSTRACT}

A dock in San Antonio, Paraguay, used to unload Liquefied oil gas (LOG) was struck by a barge during maneuvering operations. The knocked element (a pillar) was fractured and other symptoms were also noted in contiguous elements, such as cracks and deformations. A total loss was claimed to the insurance company, which led to a further investigation. The survey analysis performed required the input from an expert in engineering, knowledgeable in materials, structures and pathology of constructions. The aim of this report was to determine the real effects of the impact on the structure towards the definition of responsibilities of the parts involved. The report included the diagnosis and recommendations for the repairing, strengthening and maintenance of the structure. The verdict indicated that only a fraction of the symptoms were attributable to the impact, as the structure shows signs of other unrelated problems.

Key words: pathology; structures; surveyors; crash; durability.

Autor de contacto: Paulo G. Yugovich (yugovich@ pla.net.py) 
Revista ALCONPAT, Volumen 1, Número 2, Mayo - Agosto 2011, Páginas 135 - 148

\section{ANTECEDENTES Y OBJETO}

Teniendo en cuenta el choque de una barcaza contra un muelle portuario de descarga de GLP ubicado a orillas del rio Paraguay en el distrito de San Antonio se realizó la visita al lugar, a pedido de la liquidadora de siniestros de la aseguradora. Durante la visita se observaron tanto los daños causados por el impacto como también síntomas de fallas aparentemente relacionados con otras causas. Con base en estas observaciones se evaluaron los estudios necesarios para determinar la etiología de cada falla observada. La propietaria del muelle reclamaba el evento como siniestro total.

El documento entregado por la aseguradora para la realización del estudio consistió en una planta arquitectónica del muelle. No se disponían de los planos de detalles de armaduras ni planos de fundación de la estructura.

\section{Objeto}

El objeto del trabajo técnico era evaluar los daños causados por el impacto, discriminarlos con respecto a daños pre-existentes y determinar los trabajos necesarios de reparación para restituir la seguridad de la estructura existente antes del siniestro é incluso proponer refuerzos si fuesen necesarios.

\section{Metodología general}

La estrategia metodológica ha consistido en lo siguiente: inspección, relevamiento de datos y tomas fotográficas (no existía mucha información sobre la obra - ni siquiera edad precisa, mucho menos memoria de cálculo), ensayos sobre el hormigón de la estructura (para caracterizar el material), análisis estructural del muelle (considerando por un lado solo el peso propio y por otra parte la fuerza del choque, para entender las deformaciones y las fisuras), el diagnóstico y las recomendaciones de actuación (principalmente la reparación de los efectos del choque, pues eso definiría la cobertura de la póliza) .

\section{Inspección}

La primera visita fue de tipo general en la que se identificaron los elementos estructurales afectados directamente por el impacto. Se observó la rotura producida en un pilar impactado y fisuras en vigas ligadas a la plataforma en el sector del impacto. También se observaron deformaciones a lo largo de toda la estructura del muelle y fisuras erráticas en la superficie de la losa a lo largo de toda la pasarela. Además se observaron defectos constructivos en varios sectores como falta de recubrimientos en pilares y coqueras.

Teniendo en cuenta la falta de planos conforme a obra, las siguientes visitas fueron realizadas con el objeto de relevar las dimensiones reales del muelle y ubicar los elementos dañados en planos de encofrado. Se realizó un levantamiento topográfico planialtimétrico de toda la estructura con el objeto de modelar la estructura para el análisis estructural.

\section{TOMA DE DATOS Y REGISTROS FOTOGRÁFICOS}

De acuerdo a informaciones suministradas por funcionarios que presenciaron el siniestro, el impacto fue ocasionado por un error de maniobra de un tren con once barcazas vacías acopladas entre sí que venían subiendo desde aguas abajo. El impacto se produjo en el pilar 25 ubicado en la planta de encofrado indicada en la figura 1. 
Se realizó un levantamiento planialtimétrico, en donde se representaron los desniveles verticales y desvíos horizontales de cada punto del muelle respecto a una línea de referencia. También se definió una nomenclatura para cada elemento con la finalidad de ubicar en los planos las patologías observadas.

Las fallas patológicas observadas en el muelle se describen en la Tabla 1 y se pueden apreciar en las figuras de la 2 a la 9.
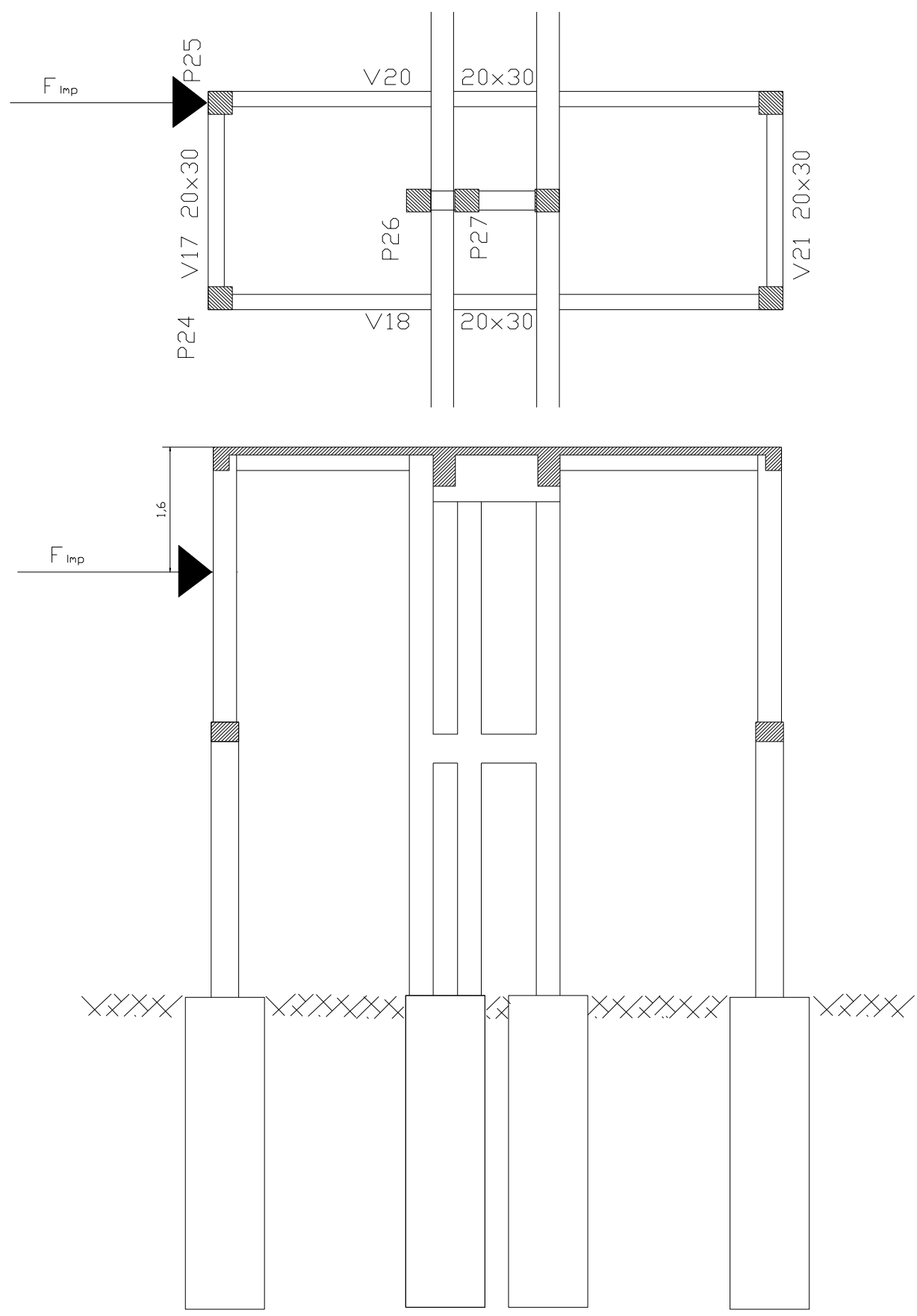

Figura 1. Punto de impacto 
Revista ALCONPAT, Volumen 1, Número 2, Mayo - Agosto 2011, Páginas 135 - 148

Tabla 1. Síntomas Patológicos observados

\begin{tabular}{|c|c|c|}
\hline ELEMENTO & TIPO DE FALLA & Figura \\
\hline PILAR 25 & Colapso en la sección transversal del pilar & 2 \\
\hline VIGA 20 & $\begin{array}{l}\text { Fisura transversal ( } 3 \text { a } 1 \mathrm{~mm} \text { ) de arriba hacia abajo } \\
\text { en sector de apoyo sobre el pilar } 30\end{array}$ & 3 \\
\hline VIGA 18 & $\begin{array}{l}\text { Fisura transversal ( } 3 \text { a } 1 \mathrm{~mm} \text { ) de arriba hacia abajo } \\
\text { en sector de apoyo apoyo sobre el pilar } 29\end{array}$ & 4 \\
\hline VIGA $1 \mathrm{~K}$ & Fisura transversal (3 a $1 \mathrm{~mm}$ ) & 5 \\
\hline LOSA 16 & Fisura diagonal ( 3 a $2 \mathrm{~mm}$ ) partiendo la la viga $1 \mathrm{k}$ & 6 \\
\hline VIGA $1 \mathrm{~J}$ & Fisura transversal $(1 \mathrm{~mm})$ & \\
\hline LOSA 18 & $\begin{array}{l}\text { Fisura transversal ( } 3 \text { a } 1 \mathrm{~mm} \text { ) en la unión con la } \\
\text { viga } 27 \text { en el nudo } \mathrm{n}^{\text {o }} 83\end{array}$ & 7 \\
\hline $\begin{array}{l}\text { PILARES } \\
9 \text { y } 10\end{array}$ & $\begin{array}{l}\text { Desprendimiento del borde externo en el nudo } \\
\text { de unión de la viga riostra con el pilar } 9 \text {. Además } \\
\text { se observa el desaplomo del pilar con un desvío } \\
\text { de } 20 \mathrm{~cm} \text { en la parte superior respecto a la base. }\end{array}$ & \\
\hline $\begin{array}{c}\text { PILARES } \\
3,4,5,6,7,8,9,10 \\
11 \text { y } 12 \\
\end{array}$ & $\begin{array}{l}\text { Se observa el desaplomo de los pilares con } \\
\text { un desvío variando de } 5 \text { a } 20 \mathrm{~cm} \text { en la parte } \\
\text { superior respecto a la base y un desnivel de } 5 \text { a } 9\end{array}$ & 8 \\
\hline $\begin{array}{c}\text { PILARES } \\
18,19,20,21,22,23,31 \\
32,33 \text { y } 34\end{array}$ & $\begin{array}{l}\text { Se observa el desaplomo de los pilares con } \\
\text { un desvío variando de } 5 \text { a } 10 \mathrm{~cm} \text { en la parte } \\
\text { superior respecto a la base y un desnivel de } 5 \text { a } 9 \\
\text { cm en la sección transversal de la pasarela }\end{array}$ & \\
\hline $\begin{array}{c}\text { LOSAS } \\
1,2,3,4,5,6 \\
7,11,12,13,14,15,16 \\
17,18,19 \text { y } 20\end{array}$ & $\begin{array}{l}\text { Fisuras erráticas en la superficie de la losa con } \\
\text { aberturas de } 0,5 \text { a } 2 \mathrm{~mm} \text { y fisuras paralelas a las } \\
\text { uniones con vigas de borde. }\end{array}$ & 9 \\
\hline $\begin{array}{l}\text { LOSAS } \\
9,10\end{array}$ & $\begin{array}{l}\text { Fisuras diagonales de } 1 \text { a } 2 \mathrm{~mm} \text { en cambio de } \\
\text { dirección de losas partiendo de encuentro con } \\
\text { pilar de borde. }\end{array}$ & \\
\hline
\end{tabular}

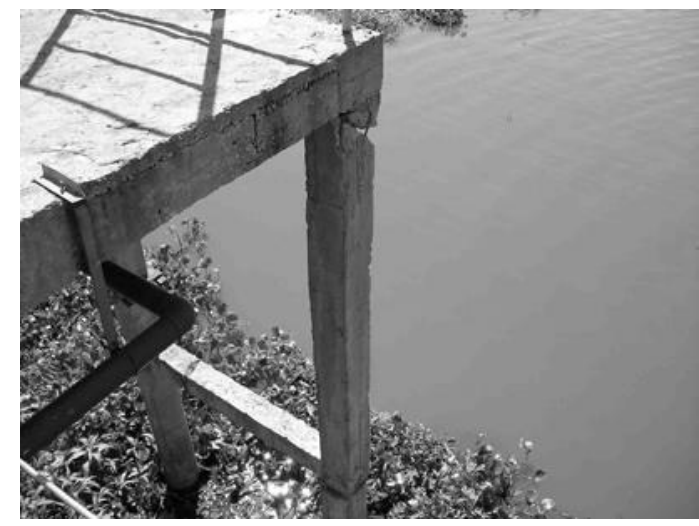

Figura 2. Pilar 25 impactado

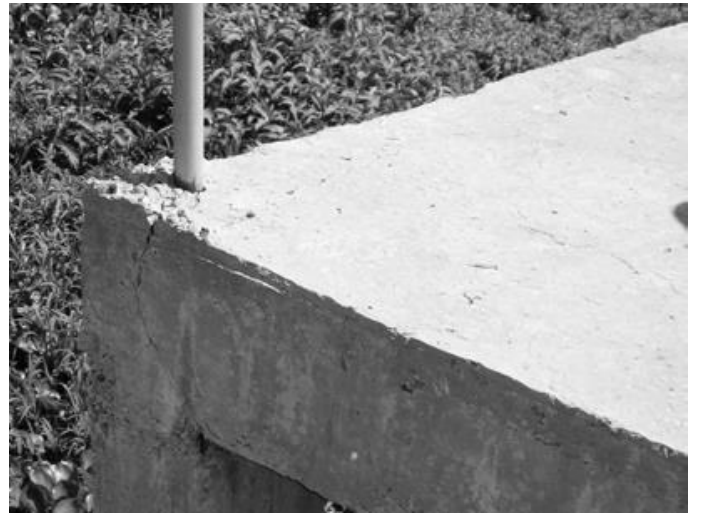

Figura 3. Viga 20 fisurada 
Revista ALCONPAT, Volumen 1, Número 2, Mayo - Agosto 2011, Páginas 135 - 148

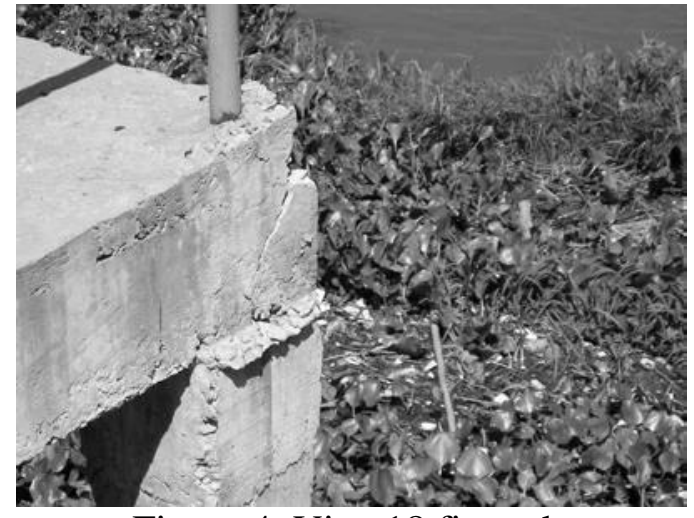

Figura 4. Viga 18 fisurada

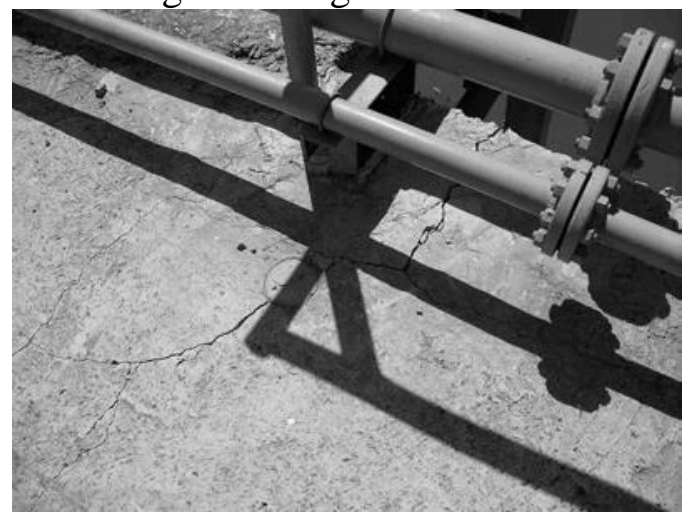

Figura 6. Losa 16 fisura diagonal

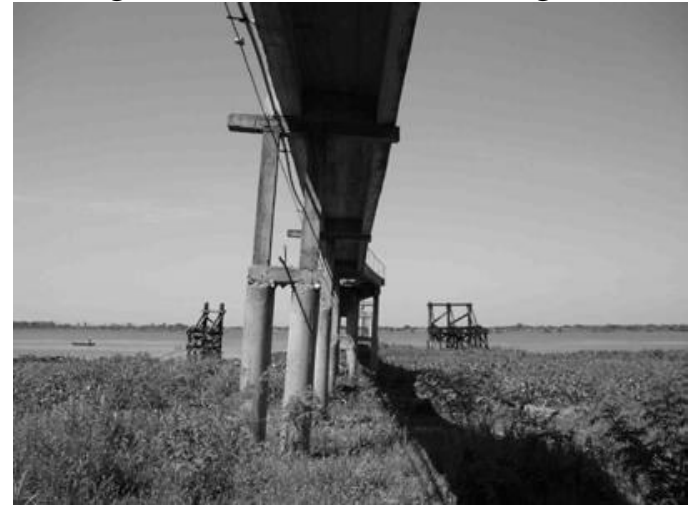

Figura 8. Pilares con desaplomo

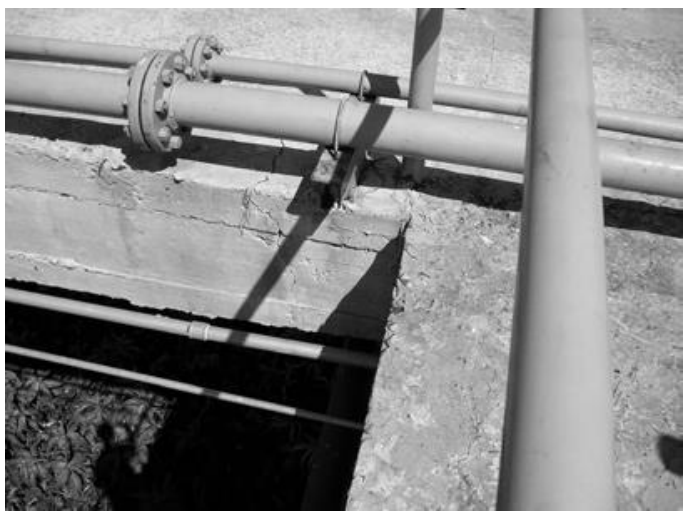

Figura 5. Viga 1k fisura transversal

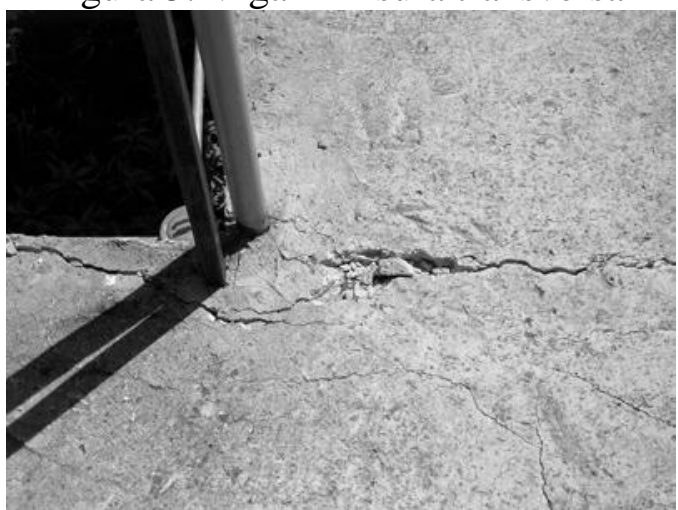

Figura 7. Losa 18 fisura transversal

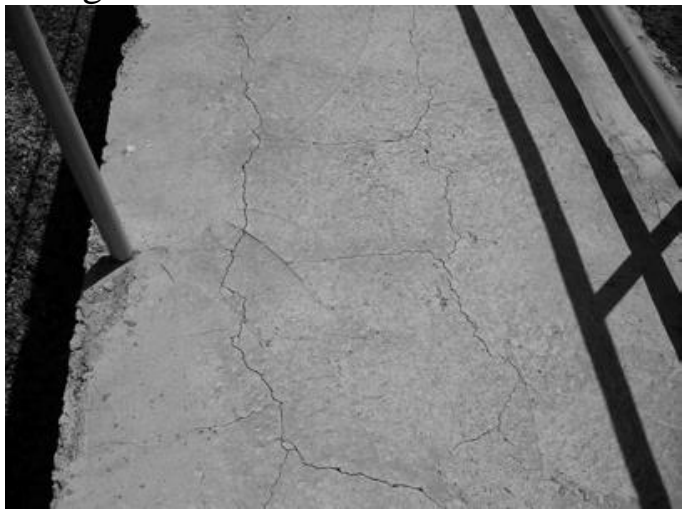

Figura 9. Fisuras en losa 
Revista ALCONPAT, Volumen 1, Número 2, Mayo - Agosto 2011, Páginas 135 - 148

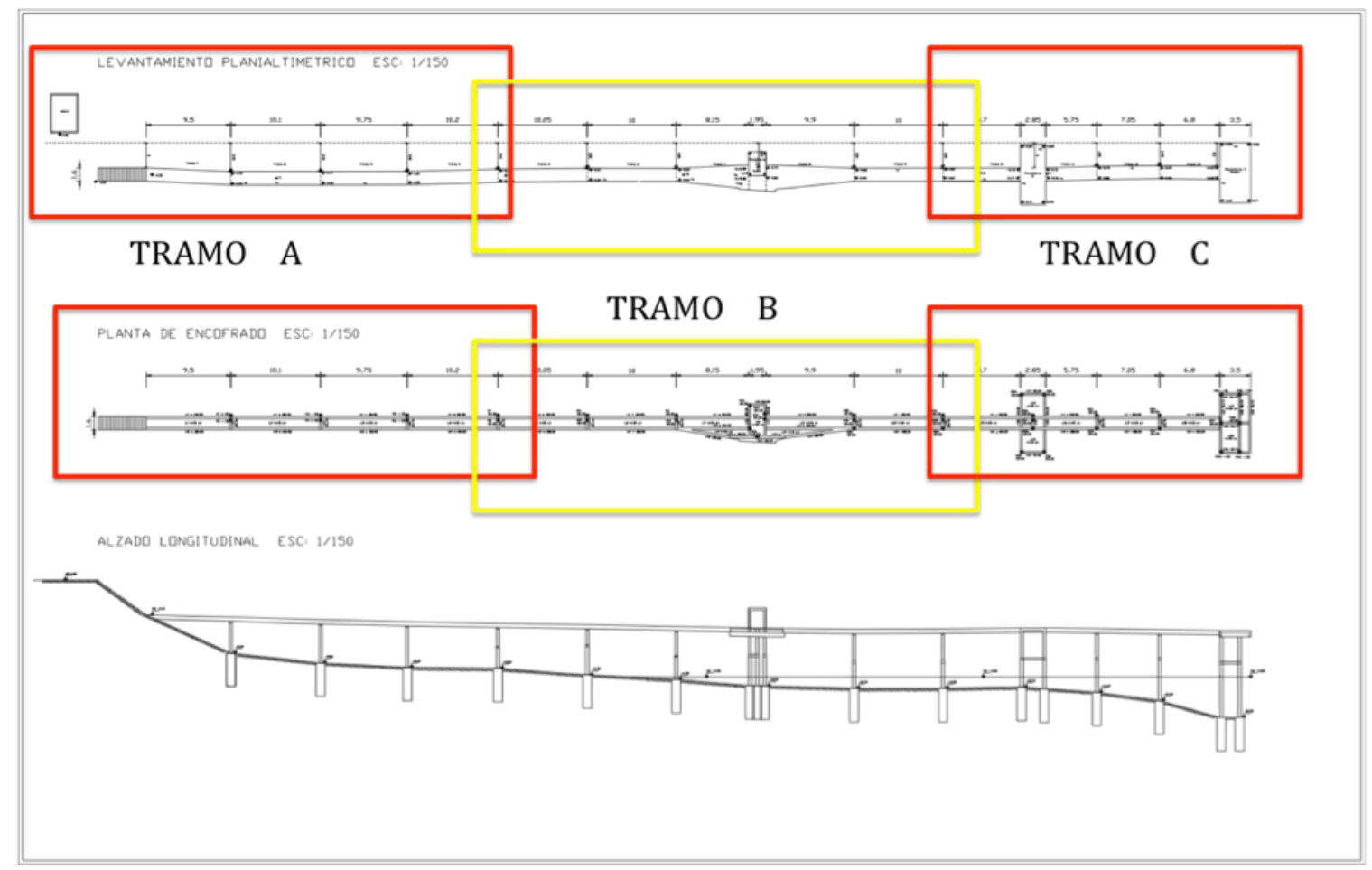

Figura 10. Planta general del muelle y alzado

\section{ESTUDIOS Y ENSAYOS REALIZADOS}

Con el objeto de determinar las propiedades mecánicas reales para verificar el comportamiento estructural, se realizaron ensayos destructivos por medio de la extracción de tres testigos "in situ" de elementos estructurales (losas) y posterior rotura de los mismos para obtener la resistencia a la compresión. También se efectuaron ensayos "no destructivos", por medio del esclerómetro de reflexión, cuyo propósito es medir el índice esclerométrico que guarda relación directa con las características resistentes y de homogeneidad del elemento estudiado.

A efectos de tratar de diagnosticar la causa de las fisuras observadas en las losas, se sacaron cuatro testigos sobre fisuras y los otros tres antes citados de lugares no fisurados para determinar luego la profundidad de carbonatación en los recubrimientos y a los costados de las fisuras (en los testigos fisurados) por medio de aspersión con solución de fenolftaleína (Figuras 11 y 12).

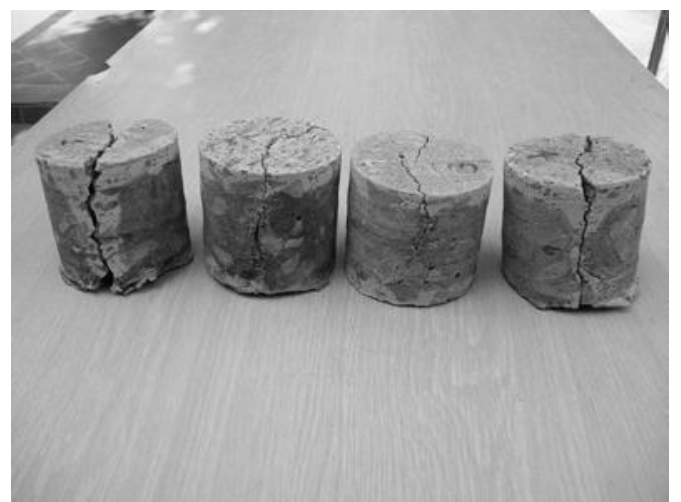

Figura 11. Testigos fisurados

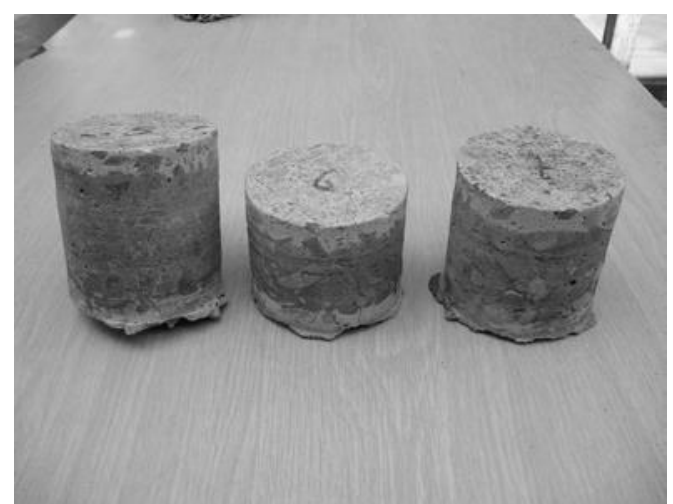

Figura 12. Testigos no fisurados 
Revista ALCONPAT, Volumen 1, Número 2, Mayo - Agosto 2011, Páginas 135 - 148

\section{RESULTADOS DE LOS ENSAYOS}

\subsection{Ensayo de compresión de testigos}

En la tabla 2 se muestra un resumen de los resultados de ensayos obtenidos en las probetas testigo.

Tabla 2. Resultados de ensayos sobre probetas testigo no fisuradas

\begin{tabular}{|l|c|c|c|}
\hline Probeta No $^{\mathbf{0}}$ & $\mathbf{5}$ & $\mathbf{6}$ & $\mathbf{7}$ \\
\hline Relación h/d & 0,95 & 0,75 & 0,85 \\
\hline Coeficiente de corrección & 0,82 & 0,70 & 0,76 \\
\hline fc Kg/cm2 & 140 & 134 & 146 \\
\hline fc corregida & 115 & 94 & 111 \\
\hline Densidad Relativa aparente & 2,55 & 2,65 & 2,56 \\
\hline Absorción de agua \% & 6,1 & 8,4 & 7,7 \\
\hline
\end{tabular}

\subsection{Ensayos esclerométricos}

Las variables estadísticas obtenidas a partir de los resultados son las siguientes:

- Valor promedio del Índice esclerométrico en losas: 30,33 (16 lecturas) Desviación Standard de los valores sn-1: 4,22

- Coeficiente de variación $\partial: 13,91 \%$

- Valor característico (95\%): 23,41

- Valor promedio del Índice esclerométrico en pilares: 35 (5 lecturas) Desviación Standard de los valores sn-1: 2,55

- Coeficiente de variación $\partial: 7,28 \%$

- Valor característico (95\%): 30,82

\subsection{Ensayos de carbonatación}

En la tabla 3 se muestran los resultados obtenido de los ensayos de carbonatación en las muestras, dichos resultados se encontraron tanto para la superficie como para las fisuras.

Tabla 3. Resultados de ensayos de carbonatación

\begin{tabular}{|c|c|c|c|}
\hline Testigo $\mathbf{N}^{\mathbf{0}}$ & Elemento & $\begin{array}{c}\text { Profundidad carbonatada } \\
\text { en la superficie }(\mathbf{c m})\end{array}$ & $\begin{array}{c}\text { Profundidad carbonatada } \\
\text { en la fisura (cm) }\end{array}$ \\
\hline 1 & Losa L16 & 1,20 & $1,00 \mathrm{a}$ \\
\hline 2 & Losa L13 & 1,10 & $0 \mathrm{a}$ \\
\hline 3 & Losa L7 & 1,00 & $0 \mathrm{a}$ \\
\hline 4 & Losa L9 & 1,50 & $0,3 \mathrm{a}$ \\
\hline 5 & Losa L7 & 1,15 & - \\
\hline 6 & Losa L5 & 1,10 & - \\
\hline 7 & Losa L2 & 1,40 & - \\
\hline
\end{tabular}

\section{CÁLCULOS REALIZADOS Y ANÁLISIS DE RESULTADOS}

A partir de los resultados de los ensayos se estimó la resistencia del hormigón a ser utilizada en la verificación del comportamiento estructural. Además se calcularon las deformaciones instantáneas 
y diferidas producidas por las diferentes hipótesis de carga combinando las acciones del peso propio de la estructura con el impacto horizontal.

De los ensayos de carbonatación en fisuras se pudo determinar el origen de las fisuras teniendo en cuenta que la profundidad carbonatada es función del tiempo de exposición y de la profundidad de la fisura. En aquellos casos en que la fisura no atraviesa totalmente la sección la profundidad carbonatada alrededor de la misma es muy inferior a aquella en que si atraviesa la sección del elemento. En este último caso se produce la infiltración de agentes externos y el lavado de los compuestos alcalinos lo que acelera la carbonatación.

\subsection{Análisis de la probable resistencia del hormigón}

En base a los resultados obtenidos en los ensayos se pudo calcular la resistencia característica estimada fc,est, la cual para muestreos pequeños y para un nivel de control normal, se obtiene a partir de la siguiente expresión (Montoya et al):

$$
\mathrm{f} \text { c,est }=\text { B.X1 }
$$

donde " $ß$ " es un coeficiente que depende del tamaño de la muestra y del coeficiente de variación del hormigón " $\partial "$ que en nuestro estudio fue obtenido a partir de los ensayos esclerométricos (ver ítem 5.2) .

En las losas este coeficiente es menor a 0,15 con lo que se concluye que la uniformidad del hormigón es buena y en los pilares es menor a 0,10 por lo que la uniformidad es excelente (Montoya et al).

Con el valor del coeficiente de variación obtenido y con el menor de los valores de resistencia, X1 $=94 \mathrm{~kg} / \mathrm{cm} 2$ se obtuvo para los tres testigos extraídos de las losas:

$$
\text { f c,est }=\text { B.X1 }=1,004 \times 94=94,38 \mathrm{~kg} / \mathrm{cm}^{2}
$$

En los pilares a partir del valor característico del índice esclerométrico en éstos y de la relación de la resistencia de los testigos obtenida en la losa con el índice esclerometrico se obtiene una resistencia característica estimada de $111 \mathrm{~kg} / \mathrm{cm} 2$.

Teniendo en cuenta que la resistencia de proyecto se verifica en base a probetas moldeadas y curadas en laboratorio se debe aplicar un coeficiente de corrección de 1,10 al valor anterior resultando:

\begin{tabular}{|c|c|}
\hline ELEMENTO & $\mathbf{f}_{\mathbf{c}, \mathrm{est}}\left(\mathbf{K g} / \mathbf{c m}^{\mathbf{2}}\right)$ \\
\hline LOSAS & 104 \\
\hline PILARES & 122 \\
\hline
\end{tabular}

\subsection{Estimación de la capacidad portante lateral de las fundaciones}

En base a informaciones de estudios de suelo SPT (Standard Penetration Test) disponibles, de las proximidades de la zona del muelle, el suelo del lugar consiste en una arcilla limosa hasta una profundidad aproximada de 4 metros a partir de la cual se encuentra una arena limosa muy densa $(\mathrm{N}=50)$. A partir de esa información se estimaron los parámetros geotécnicos para la verificación de las fundaciones. Los valores de los parámetros son:

$$
\begin{array}{ll}
\mathrm{g}_{\mathrm{sum}}=0,49 \mathrm{tn} / \mathrm{m} 3 & \begin{array}{l}
\text { (peso específico sumergido) } \\
\varnothing=0^{\circ}
\end{array} \\
\mathrm{c}=1,05 \mathrm{tn} / \mathrm{m} 2 & \begin{array}{l}
\text { (cohgulo de rozamiento interno) } \\
\mathrm{Kh}=0,19 \mathrm{~kg} / \mathrm{cm} 3
\end{array} \\
\mathrm{Kv}=20 \mathrm{~kg} / \mathrm{cm} 3 & \begin{array}{l}
\text { (coeficiente de balasto horizontal del suelo lateral) } \\
\text { (coeficiente de balasto vertical del suelo de apoyo) }
\end{array} \\
\text { P. G. Yugovich, G. A. Melgarejo }
\end{array}
$$


Considerando que las fundaciones consisten en tubulones de 1 (un) metro de diámetro (D) y que la profundidad $(\mathrm{H})$ haya llegado a los 4 metros se puede estimar la capacidad portante lateral Ep a partir de la teoría de Rankine, por la expresión:

$$
E_{p}=\gamma \cdot H \cdot \frac{H}{2} \cdot D \cdot \frac{1+\operatorname{sen} \phi}{1-\operatorname{sen} \phi}+2 \cdot c \cdot H \cdot \pi \cdot \frac{D}{2} \sqrt{\frac{1+\operatorname{sen} \phi}{1-\operatorname{sen} \phi}}=17,11 \text { ton }
$$

\subsection{Hipótesis de cargas}

Para el análisis estructural se utilizaron las siguientes hipótesis de cargas:

1.- Peso Propio de la estructura;

2.- Peso propio + Carga de impacto lateral (Figura 1)

Con la hipótesis 1 se determinaron los efectos del peso propio en las deformaciones diferidas y esfuerzos entre los pilares 3 y 12 principalmente. En la hipótesis 1 no se consideró la sobrecarga de uso teniendo en cuenta que se analizarán las deformaciones por cargas de larga duración. Por otro lado, con la hipótesis 2 se determinaron los esfuerzos y deformaciones instantáneas en los elementos impactados como también los esfuerzos transmitidos por el impacto al sector entre los pilares 3 y 12.

\subsection{Estimación de la carga de impacto}

Teniendo en cuenta la incertidumbre respecto a las variables involucradas en este análisis se determinó la carga de impacto a partir de los daños observados en los elementos directamente afectados calculando los esfuerzos admisibles de sus secciones.

Introduciendo la geometría, las secciones y las propiedades de los materiales del muelle en un modelo tridimensional generado por computadora se aumentó progresivamente la carga horizontal en el punto de impacto hasta alcanzar los esfuerzos admisibles en aquellas secciones afectadas. Como resultado se obtuvo una carga horizontal de 25 toneladas aplicada en el pilar 25 a 1,60 metros por debajo de la superficie de la plataforma 2 (ver Figura 1).

\subsection{Análisis estructural}

A partir de los datos geométricos, secciones y propiedades de los materiales obtenidos se generó un modelo tridimensional de la estructura del muelle por computadora (programa RAM advance). Los resultados del análisis estructural para las dos hipótesis permiten representar las figuras de la estructura deformada cuando actúa el impacto (hipótesis 1 - Figura 11) y cuando actúa solamente el peso propio (hipótesis 2 - Figura 12). A partir de los valores de los esfuerzos M (momento flector) y Q (esfuerzo cortante), transmitidos a las fundaciones, determinados por el cálculo, se verifican los valores de las resultantes E1 de presiones horizontales en el suelo, generados por el impacto, que se calculan por las expresiones:

$$
\begin{aligned}
& E_{1}=p_{1} \cdot \frac{H}{2} \cdot D \\
& p_{1}=\frac{\left(K_{h} / K_{v}\right) \cdot(M+Q \cdot H) \cdot H}{\frac{\pi \cdot D^{4}}{64}+4 \cdot\left(K_{h} / K_{v}\right) \cdot \frac{D \cdot H^{3}}{12}}
\end{aligned}
$$

Los valores de las resultantes de presiones horizontales transmitidas son resumidos en la tabla 4. 
Revista ALCONPAT, Volumen 1, Número 2, Mayo - Agosto 2011, Páginas 135 - 148

Tabla 4. Hipótesis 2 - Esfuerzos transmitidos al cimiento y empuje resultante

\begin{tabular}{|c|c|c|c|c|c|}
\hline Elemento & Nudos & $\begin{array}{c}\text { Esf. Normal N } \\
\text { (tn) }\end{array}$ & $\begin{array}{c}\text { Esf. Cortante } \\
\mathbf{Q} \text { (tn) }\end{array}$ & $\begin{array}{c}\text { Mom. Flector } \\
\text { Mf (tn.m) }\end{array}$ & E1 (tn) \\
\hline P20 & 39 & 16,12 & 0,77 & $-1,29$ & 1,32 \\
\hline P23 & 51 & 20,82 & 1,43 & $-2,46$ & 2,46 \\
\hline P24 & 98 & 2,10 & 0,82 & $-3,29$ & 1,98 \\
\hline P25 & 100 & 9,62 & 5,15 & $-9,33$ & 9,04 \\
\hline P29 & 99 & 6,54 & $-1,00$ & $-3,48$ & 2,26 \\
\hline P30 & 101 & 6,69 & 1,05 & $-3,61$ & 2,36 \\
\hline P26 & 78 & 1,70 & 1,55 & $-2,60$ & 2,65 \\
\hline P28 & 79 & 25,32 & 2,13 & $-3,66$ & 3,68 \\
\hline P31 & 56 & $-8,00$ & 1,85 & $-3,88$ & 3,40 \\
\hline P32 & 57 & 19,38 & 1,85 & $-3,88$ & 3,40 \\
\hline P33 & 64 & $-0,25$ & 0,90 & $-2,29$ & 1,77 \\
\hline P34 & 65 & 14,18 & 0,90 & $-2,29$ & 1,77 \\
\hline
\end{tabular}

Se observa que los valores de empujes son sensiblemente menores a los calculados para el empuje pasivo en condiciones teóricas (ver ecuación (2)). Sin embargo se ven deformaciones en la realidad. Es probable entonces que las fundaciones no hayan llegado a suelo resistente.

\section{DIAGNÓSTICO}

A partir de los análisis realizados se puede diagnosticar los siguientes aspectos:

a) Respecto a los daños observados en el pilar $n^{\circ} 25$ se puede diagnosticar con base en los valores de los esfuerzos cortantes y flectores originados por el impacto que los daños son irreparables y es muy probable que la sección inferior del pilar en la unión con el apoyo sobre la fundación bajo el agua esté dañada teniendo en cuenta las dimensiones del pilar.

b) Respecto a los daños observados en la vigas 20,18 y $1 \mathrm{k}$, se puede diagnosticar con base en los esfuerzos de flexión y a la configuración de las fisuras que los daños fueron originados por el impacto y son reparables.

c) Respecto a los daños observados en la losa L16 considerando que el ensayo de carbonatación en el testigo $\mathrm{n}^{\circ} 1$ extraído sobre la fisura demuestra que la fisura era preexistente es probable que haya sido causada por retracción hidráulica del hormigón teniendo en cuenta que se encuentra en las proximidades de la unión con la estructura de la plataforma 2. Con el impacto esta fisura pudo propagarse a consecuencia de las tensiones cortantes por torsión transmitidas por la viga $1 \mathrm{~K}$ aumentando su abertura. Este daño es reparable.

d) Respecto a los daños observados en la viga $n^{\circ} 1 \mathrm{~J}$ cerca del nudo $n^{\circ} 66$ con la viga $n^{\circ} 18$ se puede diagnosticar en base a los esfuerzos obtenidos y a la configuración de la fisura que no fue causada por el impacto. La causa probable de dicha fisura es la retracción hidráulica del hormigón.

e) Respecto a los daños observados en la losa $n^{\circ} 18$ en la unión con la viga $\mathrm{n}^{\mathbf{0}} 27$ en el nudo $\mathrm{n}^{\circ}$ 83 se puede diagnosticar en base a los esfuerzos obtenidos y a la configuración de la fisura que pudo haber sido causada por las tensiones de tracción causada por el impacto. Este daño es reparable.

f) Respecto a los daños observados en la viga riostra de los pilares $\mathrm{n}^{\circ} 9$ y 10 y a la deformación observada, se puede diagnosticar en base a la diferencia entre los esfuerzos obtenidos en las hipótesis 1 y 2 que los daños y las deformaciones observadas no fueron causados por el impacto. 
Las deformaciones observadas se deben a deformaciones diferidas bajo la acción de la cargas de peso propio de la estructura incrementadas por la baja calidad del hormigón demostrada en los ensayos de testigos y vicios constructivos como la mala compactación del hormigón observada en muchos lugares de la construcción.

g) Respecto a las deformaciones en la plomada vertical y al desnivel en la sección transversal de la pasarela observadas en la línea de los pilares $n^{\circ} 18,19,20,21,22,23,31,32$, 33 y 34, se puede diagnosticar en base a los esfuerzos transmitidos a la fundación que las deformaciones observadas pudieron ser causadas parcialmente por el impacto teniendo en cuenta que las tensiones de compresión transmitidas por el impacto a los pilares de aguas abajo aumentaron en más del $100 \%$ en comparación a la carga de servicio. Por otro lado, no existe documentación que compruebe que durante la etapa constructiva de las fundaciones se haya llegado a los estratos más duros de suelo razón por la que se pone en duda la capacidad portante real a las cargas de compresión vertical de los tubulones. Estos efectos se pueden visualizar en la figura 13, pues la deformada causada por la fuerza del choque manifiesta que la colisión ha sido responsable de varios de los síntomas patológicos.

h) Respecto a las deformaciones en la plomada vertical observadas en la línea entre los pilares $\mathrm{n}^{\mathbf{0}} 3$ a 12, se puede diagnosticar en base a la diferencia entre los esfuerzos obtenidos en las hipótesis 1 y 2 que los daños y las deformaciones observadas no fueron causados por el impacto. Las deformaciones observadas se deben a deformaciones diferidas bajo la acción de la cargas de peso propio de la estructura incrementadas por la baja calidad del hormigón demostrada en los ensayos de testigos y vicios constructivos como la mala compactación del hormigón observada en muchos lugares de la construcción. La deformada de la figura 14 muestra que las deformaciones por peso propio son importantes en los sectores en que la plataforma no está convenientemente centrada sobre los soportes, pero no tienen incidencia en la zona del impacto.

i) Respecto a las fisuras observadas en la superficie de las losas $n^{\circ} 1$ al 20 de la pasarela, se puede diagnosticar en base a los testigos $n^{\circ} 2$ y 3 que se deben a la retracción hidráulica del hormigón teniendo en cuenta que las fisuras atraviesan toda la sección, y empiezan en la superficie de la losa y se cierran hacia la cara inferior. En la superficie de la losa existe la mayor pérdida de humedad por evaporación que pudo ser causada entre otros factores por el mal curado. Esa pérdida de humedad produce un cambio de volumen de la masa del hormigón que genera tensiones en todas las direcciones dando una configuración errática a las fisuras de este tipo (Retracción plástica). Las fisuras del estado endurecido causadas por retracción hidráulica tienen patrones más regulares de perpendicularidad a las dimensiones mayores. La baja calidad del hormigón demostrada por los ensayos de compresión y absorción de los testigos refuerza esta hipótesis (relación a/c elevada).

j) Respecto a las fisuras diagonales observadas en la superficie de las losas $\mathrm{n}^{\circ} 9$ y 10 de la pasarela alrededor de la plataforma 1, se puede diagnosticar en base al testigo $\mathrm{n}^{\mathbf{0}} 4$ que se deben a la retracción hidráulica del hormigón teniendo en cuenta la profundidad carbonatada alrededor de la fisura. La configuración en sentido diagonal hacia los pilares de soporte de la plataforma se deben al cambio de dirección del eje de la estructura alrededor de esos pilares lo que induce la fisura de retracción en el sentido de la línea que une el pilar con el vértice del borde donde cambia la dirección la losa. Además la losa paralela inferior que sirve de base al motor de la bomba no presenta ninguna fisura en esa posición con lo que se demuestra que la fisura no tiene su origen en las tensiones generadas por el comportamiento estructural del muelle. 


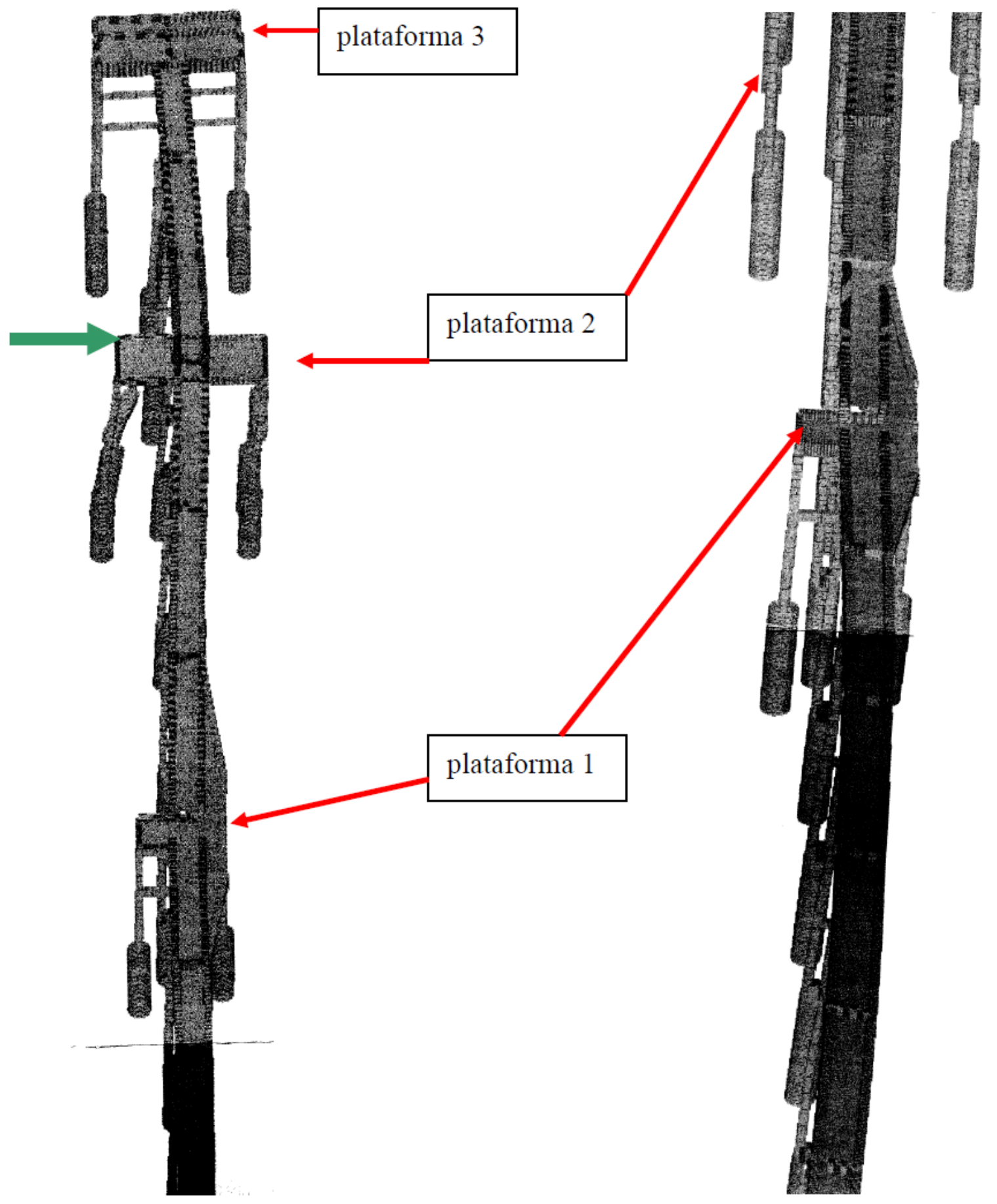

Figura 13. Deformada por choque

Figura 14. Deformada por peso propio

\section{RECOMENDACIONES DE ACTUACIÓN}

A partir del diagnóstico de los daños observados se pueden hacer las siguientes recomendaciones con el objeto de devolver a la estructura la seguridad existente antes del siniestro:

a) Debido a los daños irreparables se recomienda demoler totalmente el pilar $n^{\circ} 25$ hasta el empotramiento en el fondo y reconstruirlo asegurando la unión con la riostra horizontal y con la losa de la plataforma 2 dejando libre la armadura de los elementos existentes mediante demolición de los mismos hasta los límites del encofrado del nuevo pilar. Se deberán usar adhesivos epoxi 
(que cumplan el requisito de adherencia de la Norma ASTM C-881) para garantizar la unión entre el hormigón nuevo y el existente.

b) Los daños en el nudo de la viga $n^{\circ} 20$ con el pilar $n^{\circ} 30$ se pueden reparar mediante inyección de resina epoxi de alta fluidez (que cumple con la Norma ASTM C-881; Tipo I - Grado 1 - Clase $\mathrm{B}+\mathrm{C}$ ) en la fisura con la finalidad de restituir el monolitismo al nudo teniendo en cuenta que la deformación de la armadura superior en ese punto no afecta la seguridad estructural.

c) Los daños en el nudo de la viga $n^{0} 18$ con el pilar $n^{0} 29$ y en la viga $n^{\circ} 1 \mathrm{k}$ se pueden reparar mediante inyección de resina epoxi en la fisura con la finalidad de restituir el monolitismo al nudo teniendo en cuenta que la deformación de la armadura superior en ese punto no afecta la seguridad estructural.

d) Los daños observados en la losa L16 y L18 se pueden reparar mediante inyección de resina epoxi.

e) Las deformaciones en la pasarela y los pilares de apoyo $\mathrm{n}^{\mathrm{o}} 18,19,20,21,22,23,31,32,33$ y 34 son irreversibles. Teniendo en cuenta que no se sabe si la fundación es estable bajo las condiciones actuales se recomienda el refuerzo de los vanos con nuevos pilares fundados en tubulones intermedios que lleguen a una profundidad mínima de 4 metros. Las uniones con las pasarelas se deberá hacer hormigonando las nuevas vigas riostras con las vigas existentes de la pasarela, previo picado localizado del hormigón de las mismas, y utilizando adhesivo epóxico para unir hormigón nuevo con el viejo.

Como el objetivo principal del trabajo era diferenciar el efecto causado por el choque de la barcaza con relación a la totalidad de los problemas observados, algunos pre-existentes y relacionados a durabilidad, con el fin de acotar la responsabilidad de la empresa aseguradora, no se ha profundizado en el estudio de los problemas de corrosión salvo lo mínimo relacionado a los recubrimientos, carbonatación y compactación del hormigón. Pero los autores, aprovechando el estudio, han realizado recomendaciones generales para el mantenimiento correctivo y preventivo de la estructura en lo relacionado a la durabilidad, aparte de las específicas de rehabilitación y refuerzo estructural.

\section{CONCLUSIONES}

En base al análisis de los resultados obtenidos en los ensayos y a los cálculos realizados se puede concluir que los síntomas patológicos observados en los elementos estructurales en el tramo entre la escalera y la plataforma 1 se deben en la mayoría de los casos a vicios de las etapas de proyecto y de construcción. Las deformaciones y algunas fisuras observadas entre el tramo 8 y la plataforma 3 del muelle ya presentan influencia del impacto ocurrido, independientemente de que pudiera haber tenido también deformaciones previas al impacto, como aparece en el análisis estructural con el peso propio.

Cabe resaltar que la baja calidad del hormigón, la falta de recubrimiento de armaduras, los nidos o coqueras por mala compactación del hormigón, las fisuras de retracción en la superficie de la pasarela y los problemas de corrosión localizados, son vicios de proyecto y/o constructivos que afectan la durabilidad de la estructura a corto y mediano plazo, y ya han producido daños observables a simple vista, y previsiblemente desencadenarán nuevas patologías estructurales que disminuirán la serviceabilidad del muelle.

Respecto al diseño de la estructura, cabe resaltar la necesidad de construir estructuras de defensa para el muelle con el objeto de evitar futuros accidentes garantizando la seguridad de la misma teniendo en cuenta que se descargan materiales inflamables durante su operación. La carencia de estas defensas se considera una falla de proyecto. 
Es evidente que muchos síntomas observados en la estructura, los cuales disminuyen la serviceabilidad de la misma, así como su vida útil de servicio, son anteriores al siniestro, y al respecto recomendamos considerar seriamente la posibilidad de hacer trabajos mucho mayores para restituir seguridad al muelle ó incluso analizar la posibilidad de demoler todo y realizar un muelle nuevo considerando en el proyecto todos los factores necesarios, un proceso constructivo correcto y materiales apropiados para tener una estructura segura y durable (Red Rehabilitar).

\section{REFERENCIAS}

Jiménez Montoya P., García Meseguer A., Morán Cabré J., 12ª . Edición, Hormigón Armado, Tabla 9.4.

Red Rehabilitar, Editores Paulo Helene, Fernanda Pereira, Manual de Rehabilitación de Estructuras de Hormigón (Reparación, Refuerzo y Protección). 